programmes, non-members are not precluded from taking part in exchanges. International exchanges will no doubt be arranged, too, and the London Zoo has made progress in this direction.

The committee will also support efforts of member zoos towards conservation of species in a wider sense. It will keep a register of all rare mammals and birds kept by member zoos with special emphasis on those species considered to be in danger of extinction in the wild. Zoos will be encouraged to make every effort to breed the rare ones in captivity so that in the future there should be less demands on the depleted wild populations. In some cases zoo-bred animals may be used to reinforce those wild populations which are in danger of complete extinction. This means where practicable the controlled release of captive bred specimens in suitable reserves in their native land. Eagle owls have been sent to Sweden from the Norfolk Wildlife Park for release to augment the native population. This type of co-operation can have beneficial side-effects. For example, thirty Swinhoe's pheasant were sent from Britain to Taiwan where they were released. The Taiwan Government became interested in the project, and as a result it has given twenty-seven species legal protection in the country. Member zoos have in their collections ninety-three species of mammals and thirty-two species of birds totalling 1,194 specimens which are either on the IUCN/ICPB Red Data Book list of endangered species or which are considered likely to become rare in the wild in the future. Among rare species being bred in member zoos are the chimpanzee, the Formosan Sika deer, the Arabian gazelle and several species of pheasant.

\section{Cholera and WHO}

\section{from our Microbiology Correspondent}

THe unexpected spread from the Celebes in the early 1960s of the El Tor cholera vibrio in epidemic proportions reawakened concern and interest in cholera, a disease which, in the previous fifteen years, had shown signs of declining. A collaborative programme on cholera research was established by a joint PhilippinesJapan-WHO Cholera Committee, the chief aims of which were to evaluate the effectiveness of various cholera vaccines and to gain information on cholera carriers, the survival of the causal vibrio in natural environments and the value of various types of therapy. The results of this large scale study, published in the current issue of the Bulletin of the World Health Organization $\left(3^{1 \%}, 697 ; 1967\right)$, represent a major contribution to the understanding of this particular communicable disease.

The first two reports discuss the results of cholera vaccine preparation and field trial. Azurin and his colleagues conducted controlled field trials in the Philippines and found that a single vaccination with routine fluid vaccines (either classical Vibrio cholerae or El Tor) produced only short termed immunity of about two months. This contrasted with a reasonably high protection conferred by a $V$. cholerae oil-adjuvant vaccine for a duration of six months. The disadvantage of the oil-adjuvant vaccine, however, was its propensity to cause severe vaccination reactions in a significant proportion of the population and consequently its use was not recommended. If cholera is to be controlled primarily through vaccination programmes, further assessment of existing and new preparations is imperative. A group at the Serum Institute in Chiba (Ogonuki, Hashizume and Takashashi) discuss the results of their work on oil-adjuvant vaccines. Laboratory tests of this type of vaccine indicated high potency. while volunteer trials confirmed the enhanced protec. tive effect. Unfortunately, the vaccine reaction also was confirmed and found to be caused by local hypersensitivity and foreign-body reactions. Until quite recently it was widely held that antibiotic therapy could not affect cholera prognosis. Subsequently, the validity of chemotherapy as a supplementary treatment for cholera was recognized and has been used to arrest diarrhoea and reduce the incidence of vibrios excreted in the faeces. Kobari et al. investigated the effect of tetracycline, chloramphenicol, erythromycin and kanamycin, administered orally or intravenously. on the course of El Tor cholera. Antibiotic therapy produced a dramatic reduction in the vibrio count in stools within one hour, and after ten hours a complete disappearance of vibrios had occurred. Antibiotics such as kanamycin which are not absorbed were the least effective in treating the disease, even when doses were frequent; this results reflects the short residence time of the drug in the intestines of cholera patients. The intravenous injection of adsorbable antibiotics may prove to be the most efficient since, in the cholera condition, large volumes of body fluids are passed into the intestines and the drug may be present at its site of action in concentrations higher than those resulting from oral administration. The results of case histories appear to substantiate this latter suggestion.

The joint Cholera Committee also investigated cholera carriers in the Philippines. A carrier rate of nearly 22 per cent was found among household contacts of cholera patients, 8 per cent in immediate neighbours but only one-third per cent in the general population. The carrier state appeared to last from five to nineteen days and vibrio strains found in such carriers were identical with strains isolated from chronic sufferers. Perhaps the most significant finding in this study was that of a long-term cholera carrier ("Cholera Dolores"), the first ever to be described. The carrier contracted El Tor cholera in 1962 and continued intermittent excretion of the vibrio up to the time that Azurin and his co-workers reported in the WHO Bulletin. Strain characteristics of the vibrio and immunological evidence confirmed that this was a case of a chronic carrier and not of reinfection. In this instance the causal bacterium produced a reservoir in the gall bladder and/or biliary tract. The authors point out that although "Cholera Dolores" has not been incriminated epidemiologically - almost certainly reflecting the immunity of her family and contacts-the impact of her presence in a non-endemic area could be vastly different. Not unreasonably the analogy is drawn with the classic chronic carrier "Typhoid Mary". Also important in this context are the findings from basic and applied studies on El Tor viability and the occurrence of drug-resistant strains. Prompt and thorough disinfection of contaminated materials is clearly necessary in view of the long period of survival of El Tor vibrios under suitable conditions. Similarly, the discovery of a natural multiple-antibiotic-resistant strain that could transmit its resistance via conjugation to sensitive enteric bacteria such as Salmonella and Shigella must be held up as a further caution to indiscriminate chemotherapy. 